$>$ Les douleurs musculaires ou myofasciales en relation avec une dystrophie musculaire peuventelles être soulagées par une acupuncture centrée sur le muscle? Après un rappel sur les notionsclés de «trigger points myofasciaux» et de manipulations spécifiques des aiguilles d'acupuncture, nous rapportons quelques cas cliniques complexes parmi les patients atteints de maladies musculaires (MNM) et de la maladie de Charcot Marie Tooth (CMT) chez qui les douleurs myofasciales n'ont pas pu être contrôlées par des traitements médicamenteux et/ou par des techniques physiques. Ces patients ont été vus en consultation externe entre 2010 et 2017 à l'hôpital Rothschild, dans le service de Médecine Physique et de Réadaptation (MPR), en lien avec le Centre de Référence neuromusculaire «ParisNord/Est/Île-de-France ». Après la consultation initiale, plusieurs protocoles de soins ont été proposés, parmi lesquels figurait l'acupuncture effectuée par un médecin MPR. <

\section{Douleurs musculaires dans les dystrophies musculaires}

Dans les maladies neuromusculaires, c'est l'unité motrice qui est affectée. Ce sont par définition des affections dans lesquelles les éventuels troubles sensitifs et douloureux sont au deuxième plan. Pourtant, la fréquence des phénomènes douloureux ainsi que leur ressenti négatif ont été souvent rapportés.

Dans une étude [1] réalisée chez 68 patients adultes vus en consultation multidisciplinaire pour une myopathie héréditaire, 46 patients (soit $67 \%$ des patients interrogés) souffraient de douleurs chroniques, incluant 16 dystrophies myotoniques de types 1 et 2 ; 3/4 myopathies congénitales; $6 / 9$ myopathies métaboliques; $1 / 2$ myopathies non étiquetées).

D'après une enquête réalisée auprès de 511 patients MNM, commanditée par l'AFM-Téléthon et menée par l'équipe du Centre Anti-Douleur du CHU Saint-Antoine

\section{Acupunture et douleurs \\ musculaires dans les dystrophies musculaires}

Quels effets?

\author{
Patrick Sautreuil $^{1}$, Tuy Nga Brignol ${ }^{2}$, \\ Philippe Thoumie ${ }^{1}$
}

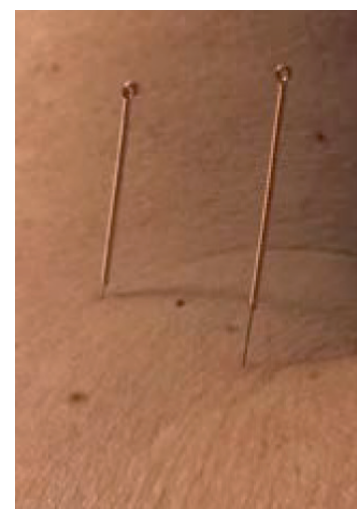

${ }^{1}$ Service de Rééducation neuro-orthopédique, Hôpital Rothschild, Paris, France. ${ }^{2}$ Association Française contre les Myopathies (AFM-Téléthon), Évry, France.

patrick2sautreuil@gmail.com

(APHP) [2], $67 \%$ ont rapporté des phénomènes douloureux dans les trois mois qui ont précédé l'enquête. L'intensité était en moyenne de $4,8 / 10$ et les douleurs étaient plus souvent intermittentes que continues. Ce travail souligne l'importance du phénomène et incite à mieux évaluer la douleur dans la prise en charge au long cours de ce type de pathologies.

D'après une étude américaine (questionnaire sur les douleurs ressenties) menée auprès de 235 patients atteints de dystrophie musculaire facio-scapulo-humérale (FSHD) ou de dystrophie myotonique de type l (DMI), la douleur est également un symptôme fréquent. La fréquence des phénomènes douloureux est plus élevée dans la FSHD (82\%) que dans la DMI (64\%). Ces douleurs sont chroniques, d'une durée moyenne de 11 à 13 ans. Les traitements antalgiques habituellement prescrits sont globalement peu efficaces.

La douleur est préférentiellement localisée aux épaules et aux hanches chez les patients FSHD alors qu'elle se situe davantage aux extrémités (pieds, mains) chez les patients DMl [3].

\section{Prise en charge de la douleur musculaire en médecine physique et acupuncture}

Les séances d'acupuncture s'inscrivent dans une prise en charge globale en Médecine Physique. Massages, étirements et renforcement musculaire, travail de l'équilibre, marche et endurance ont des bien- 


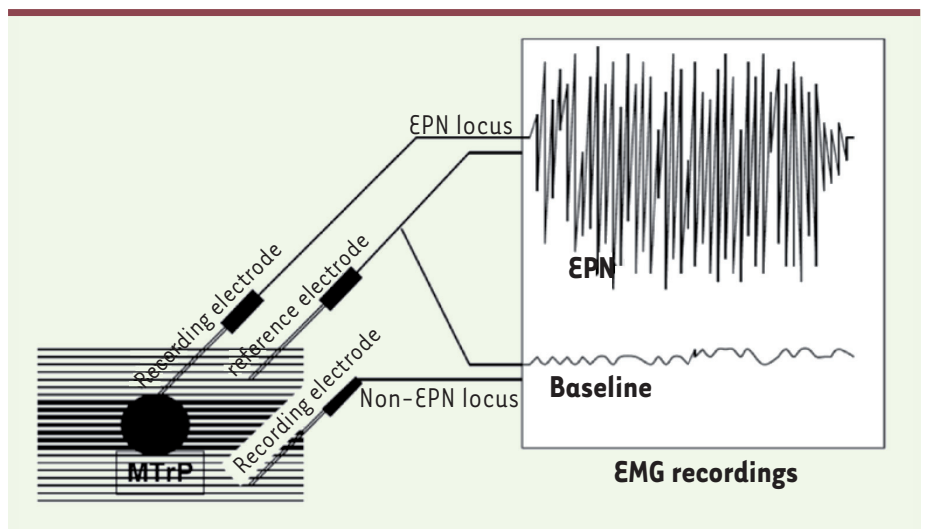

Figure 1. Au niveau du trigger point musculaire, dans le muscle au repos, l'aiguille d'électromyographie enregistre une persistance d'activité alors qu'autour l'activité électrique est nulle (EPN : End Plate Noise ; MTrP : Myofascial Trigger Point).

faits immédiats mais aussi plus tardifs, d'autant plus si le patient, au long cours, entre les séquences d’hôpital de jour, pratique régulièrement les exercices d'auto-entretien appris en rééducation. Certains trouvent également un bénéfice à la pratique régulière et complémentaire du yoga indien, du Pigong ou du Taijiquan chinois.

\section{Le concept de Trigger points}

On doit à Janet Travell et David Simons [4] le concept de trigger points myofasciaux ainsi que celui de douleurs référées ou irradiations associées. II s'agit de faisceaux musculaires plus fermes à la palpation, où la pression des doigts déclenche une douleur sourde, diffuse, irradiant parfois à distance, identique à celle dont se plaint le patient. Ces notions ont été développées initialement pour comprendre les douleurs dans les muscles du sujet sain présentant une sur-utilisation ou après traumatismes s'accompagnant de lésions musculaires. Ces deux auteurs ont également décrit une contraction vive, locale (twitch response), correspondant à une contraction musculaire à la palpation du trigger point.

On assimile maintenant ces faisceaux douloureux à la palpation des zones où persiste une contraction alors que le muscle est au repos. Cette situation est résumée dans le schéma de la Figure 1.

\section{Quelques notions d'acupuncture}

L'application de l'acupuncture dans les douleurs musculaires des dystrophies musculaires est encore peu répandue. Notre expérience acquise chez 20 patients atteints de MNM et 2 atteints de CMT suivis dans le service de Rééducation Neuro-Orthopédique de l'Hôpital Rothschild nous permet d'affirmer son intérêt à titre de complément ou à la place des traitements médicamenteux et/ou une prise en charge bien conduite en rééducation en hôpital de jour.

\section{La puncture sèche ou Dry Needling}

Le Dry Needling ou puncture sèche se différencie des traitements par injection (association anti-inflammatoires - anesthésiques). Elle ne fait pas référence à une cartographie des points d'acupuncture, contrairement à l'acupuncture chinoise traditionnelle ou énergétique. Sa pratique se répand en Amérique du Nord et en Europe.

\section{Déroulement d'une séance d'acupuncture}

La séance d'acupuncture se déroule selon les étapes suivantes:

1. Description de la ou des douleurs par le patient (évaluation selon l'échelle numérique, localisations et irradiations, réactions aux traitements).

2. Palpation digitale minutieuse pour faire la différence entre peau et espace sous cutané (palper-rouler), muscles et autres douleurs viscérales.

3. Insertion des aiguilles aux points de déclenchement de la douleur : 'loco dolenti' ou point Ashi (notion classique chinoise, qui peut se traduire par «c'est là que j'ai mal »).

4. Recherche du deqi.

La recherche du deqi correspond à une manœuvre bien connue en acupuncture traditionnelle. Elle consiste à appliquer à chaque aiguille un mouvement associant mouvement vertical et rotation alternés jusqu'à ce que l'aiguille soit «accrochée » par les tissus qu'elle traverse (Figure 2).

Initialement indolore, cette action déclenchera une douleur lentement progressive. Le praticien doit s'assurer que c'est le même type de douleur que celle ressentie de façon spontanée et à la palpation. L'obtention du deqi garantit l'efficacité de la puncture. Il se crée avec la répétition des séances un échange patient-praticien via la manipulation de l'aiguille.

Des études chez l'homme et les modèles animaux ont montré que dans la «prise d'aiguille», la composante biomécanique du de qi peut être causée par un enroulement du tissu conjonctif autour de l'aiguille [5].

Selon les circonstances, certains points d'acupuncture traditionnelle chinoise sont utilisés. Chaque séance dure environ 30 minutes.

\section{Quelques exemples de cas}

Des séances d'acupuncture ont été dispensées à des patients souffrant de FSHD, de DMI, de CMT, de myasthénie auto-immune, et de myopathie mitochondriale. Les tableaux cliniques sont très inhomogènes, y compris parmi les patients porteurs d'une même pathologie.

\section{Myopathie atypique}

Une jeune femme de 34 ans, souffrant de déficits vers l'âge de 10 ans, arthodésée Tl-L3 à l'âge 21 ans, puis désarthrodésée deux ans plus tard, souffrait depuis plus de six mois de dorsalgies gauche. La sarcopénie rendait 


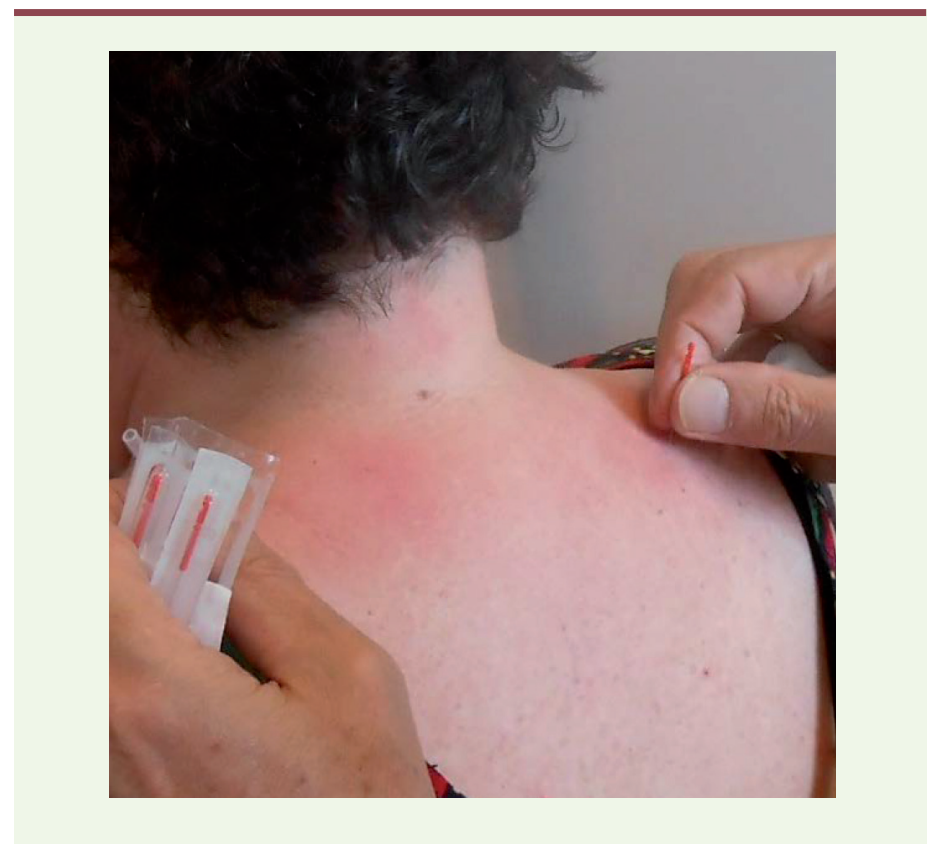

Figure 2. Puncture et recherche de deqi chez une patiente présentant des douleurs des deux épaules dans le contexte d'une myasthénie auto-immune.

la recherche des trigger points difficile mais cependant un point a été localisé à proximité de l'angle inférieur de l'omoplate gauche (Figure 3). Sa puncture répétée a progessivement amélioré le tableau clinique.

\section{Dystrophie musculaire d'Emery-Dreifuss}

Un patient âgé de 32 ans atteint de cette forme particulière de dystrophie, ayant déjà bénéficié d'une greffe cardiaque, s'est vu prescrire une statine après un bilan sanguin révélant une augmentation du cholestérol sanguin. Ce médicament a déclenché un tableau de douleurs musculaires aiguës partiellement résolutives à l'arrêt du médicament et à la prescription d'antalgiques. Des douleurs résiduelles rebelles concernaient le dos dans sa moitié gauche. Une application des aiguilles au niveau des points douloureux retrouvés à la palpation ont réduit les douleurs dès la première séance, le soulagement étant consolidé par une seconde séance (Figure 4).

\section{Dystrophie musculaire facio-scapulo-humérale}

C'est la maladie la plus représentée dans notre série (6/18). Parmi les patients, un adulte de 43 ans, dentiste, présentant une topographie classique de l'atteinte musculaire (déficit des trapèzes et des autres stabilisateurs de l'omoplate) a été vu pour douleurs. Les douleurs pouvaient atteindre 8/10 en cours d'après-midi étaient associées à une importante fatigue. L'acupuncture a été réalisée dans le contexte d'une hospitalisation de jour au cours de laquelle le patient a appris des gestes d'auto-entretien qu'il a très régulièrement réalisés. Le bénéfice de la rééducation a été indiscutable, permettant un renforcement musculaire relatif mais réel. L'effet de l'acupuncture a été jugé sur le niveau du fond douloureux qui est descendu de $5 / 10$ à $2 / 10$ sans modifier les pics liés à la fatigue qui restaient à $8 / 10$ (Figure 5).

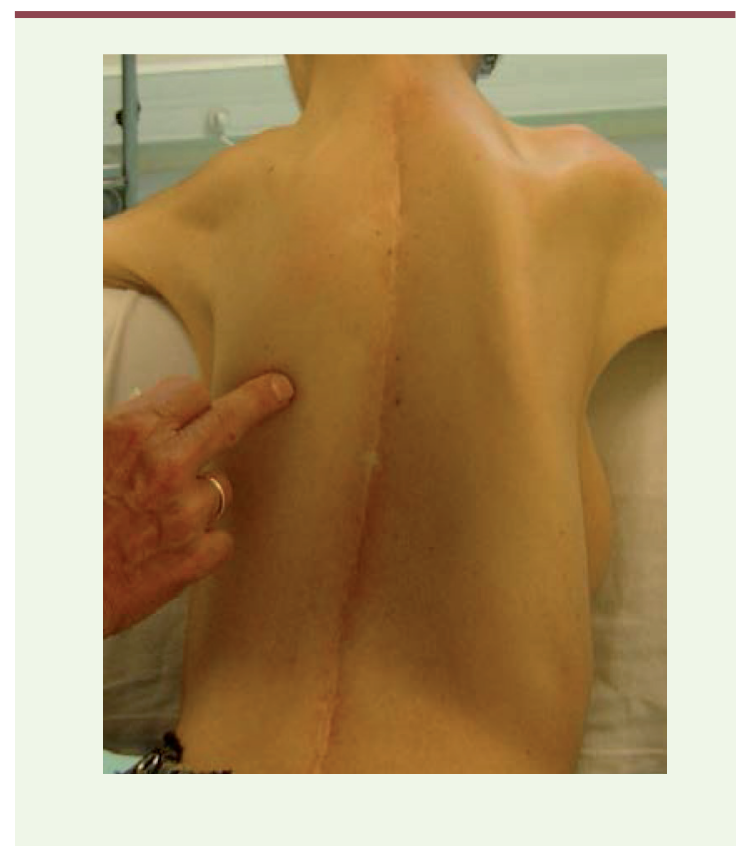

Figure 3. Palpation et découverte du trigger point responsable des dorsalgies. Recherche difficile en raison de l'amyotrophie.

\section{Discussion}

\section{Résultats}

L'efficacité de cette forme d'acupuncture est réelle comme le prouve le ressenti positif du patient: moins de douleurs, moins de fatigue, meilleure façon de se tenir debout et de marcher.

Les patients répondeurs pouvaient avoir une douleur mesurée à $8-10 / 10$ réduite à $0-2 / 10$ au décours de la séance d'acupuncture. Le bénéfice pouvait durer quelques jours à quelques semaines.

\section{Mécanismes d'action}

Les mécanismes d'action de l'acupuncture restent mal connus. La réaction à la manipulation des aiguilles d'acupuncture appelée deqi, largement considérée comme essentielle à l'effet thérapeutique de l'acupuncture, peut être une clé pour comprendre son mécanisme d'action. Deqi comprend une sensation d'aiguille caractéristique, perçue par le patient, et une «prise d'aiguille » par les tissus perçue par le médecin acupuncteur, une résistance progressive à la poursuite du mouvement de l'aiguille insérée. D'après Langevin et al [5], la prise d'aiguille est due au couplage mécanique entre l'aiguille et le tissu conjonctif avec enroulement du tissu autour de l'aiguille pendant la rotation de l'aiguille. De plus, la manipulation de l'aiguille transmet un signal mécanique aux cellules du tissu conjonctif par mécanotransduction. Cela pourrait expliquer les effets 


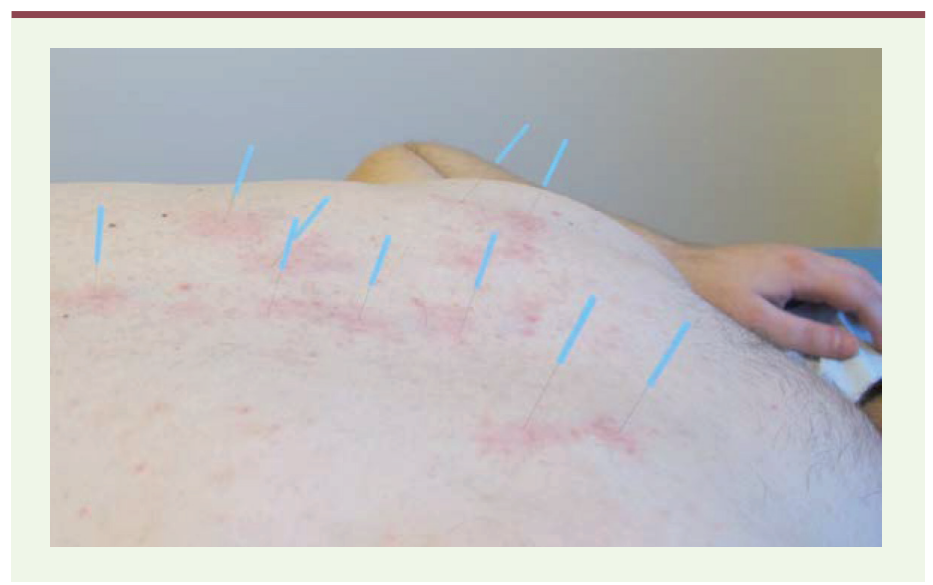

Figure 4. Patient présentant une myopathie d'Emery-Dreifuss et un tableau douloureux secondaire à la prise de statines.

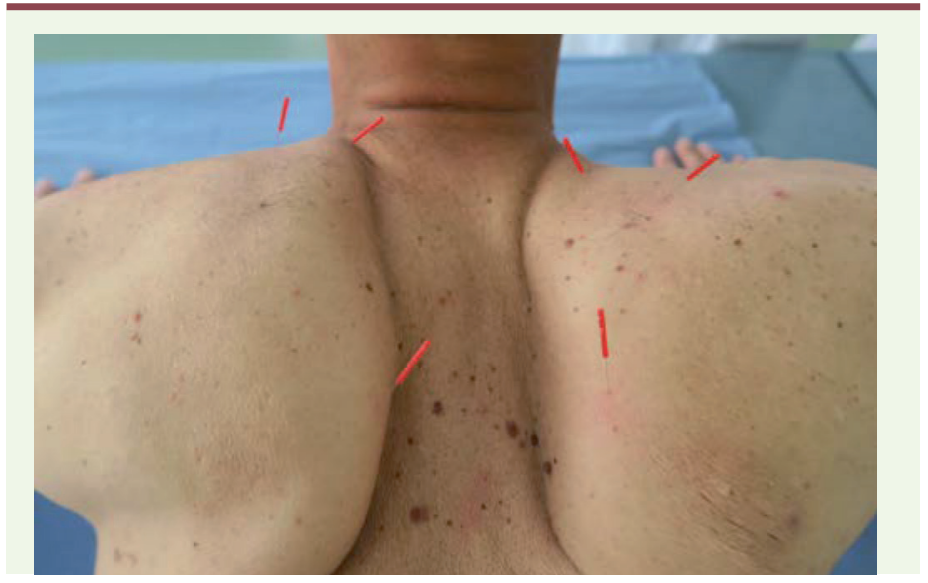

Figure 5. Puncture des trigger points des muscles trapèzes, angulaires de l'omoplate et rhomboïdes. Cliché pris pendant une séance d'acupuncture au cours d'un effort de soulèvement par poussée sur les mains. II met en évidence la faiblesse des stabilisateurs des épaules.

locaux et à distance, ainsi que les effets à long terme de l'acupuncture.

Des études sur le lapin $[6,7]$ ont montré une augmentation significative $(p<0,05)$ des taux de bêta-endorphine sérique et d'enképhaline spinale. Par ailleurs, des travaux d'imagerie cérébrale ont montré l'impact de séances d'acupuncture répétées sur la connectivité cérébrale : substance grise périaqueducale, cortex frontal médian et hippocampe bilatéral [8] Dans le traitement de la douleur chronique, l'acupuncture traite à la fois la cause de la douleur ainsi que le signal de la douleur, envoyé par la lésion tissulaire et sur la mémoire de ce signal au niveau des centres de la douleur du système nerveux central.

\section{Conclusions}

L'acupuncture offre des possibilités réelles pour traiter certaines douleurs chroniques des patients atteints de MNM : c'est une proposition de traitement physique, non médicamenteux. La détermination du point à «puncturer» réclame doigté et minutie. L'insertion des aiguilles est subtile. Leur manipulation doit provoquer le deqi ou saisie de l'aiguille par les tissus sous-cutanés. Cette forme de dialogue avec le patient via l'aiguille nécessite un apprentissage. C'est une thérapie complémentaire associée aux autres traitements. C'est une alternative, une solution différente, quand les médicaments et les traitements physiques ont montré leurs limites. Elle présente peu d'effets secondaires contrairement aux antalgiques. Elle devrait être pratiquée par davantage de médecins pour évaluer son efficacité et diversifier son utilisation. $\diamond$

Can acupuncture help relieve muscle pain in muscular dystrophy?

NB. Prise en charge : les médecins formés à l'acupuncture sont les seuls professionnels à pouvoir la pratiquer. Le remboursement de ces soins dépend du cadre d'exercice du praticien: presque complet pour les médecins en secteur I, partiel et dépendant de la mutuelle pour ceux exerçant en secteur II, il n'est pas remboursé pour ceux ayant opté pour le secteur III.

\section{LIENS D'INTÉRÊT}

Les auteurs déclarent n'avoir aucun lien d'intérêt concernant les données publiées dans cet article.

\section{RÉFÉRENCES}

1. Delorme T, Boureau F, Eymard B, et al. Clinical study of chronic pain in hereditary myopathies. Eur J Pain $2004 ; 8: 55-61$.

2. Guy-Coichard C, Nguyen DT, Delorme T, Boureau F. Pain in hereditary neuromuscular disorders and myasthenia gravis: a national survey of frequency, characteristics, and impact. J Pain Symptom Manage 2008 ; 35 : 40-50.

3. Jensen MP, Hoffman AJ, Stoelb BL, et al. Chronic pain in persons with myotonic dystrophy and facioscapulohumeral dystrophy. Arch Phys Med Rehabil $2008 ; 89: 320-8$.

4. Simons DG. Cardiology and myofascial trigger points: Janet G. Travell's contribution. Tex Heart Inst J 2003 ; $30: 3-7$.

5. Langevin HM, Storch KN, Snapp RR, et al. Tissue stretch induces nuclear remodeling in connective tissue fibroblasts. Histochem Cell Biol $2010 ; 133$ 405-15.

6. Hsieh YL, Yang SA, Yang CC, Chou LW. Dry needling at myofascial trigger spots of rabbit skeletal muscles modulates the biochemicals associated with pain, inflammation, and hypoxia. Evid Based Complement Alternat Med $2012 ; 2012: 342165$.

7. Hsieh YL, Hong CZ, Liu SY, et al. Acupuncture at distant myofascial trigger spots enhances endogenous opioids in rabbits: a possible mechanism for managing myofascial pain. Acupunct Med 2016 ; 34 : 302-9.

8. Egorova N, Gollub RL, Kong J. Repeated verum but not placebo acupuncture normalizes connectivity in brain regions dysregulated in chronic pain. Neuroimage Clin 2015 ; 9 : 430-5. 